\title{
Investigation of the Specific Energy Absorption of Triply Periodic Minimal Surfaces Subjected to Impact Loading
}

\author{
Sara AlMahri ${ }^{1, *}$, Rafael Santiago ${ }^{1}$, Dong-wook Lee ${ }^{1}$, Henrique Ramos ${ }^{1,2}$, Haleimah \\ Alabdouli ${ }^{1}$, Mohamed Alteneiji ${ }^{1}$, Zhongwei Guan ${ }^{1}$, and Marcilio Alves ${ }^{2}$ \\ ${ }^{1}$ Advanced Materials Research Centre, Technology Innovation Institute, Abu Dhabi, United Arab \\ Emirates \\ ${ }^{2}$ Department of Mechatronics and Mechanical Systems Engineering, University of São Paulo \\ (USP), São Paulo, Brazil
}

\begin{abstract}
Triply periodic minimal surfaces (TPMS) have attracted tremendous research interest due to their lightweight and superior mechanical properties. In this study, two TPMS sheet-based structures (FRD and Neovius) are designed, fabricated, and tested under dynamic and quasistatic loading conditions. Selective laser melting (SLM) is employed to facilitate the fabrication of such complex structures out of stainless steel (SS316L). Scanning electron microscopy (SEM) is utilized to assess the quality of the printed structures. The dynamic compressive behaviour is investigated through performing a direct impact compression test via a Direct Impact Hopkinson Bar (DIHB) at a strain rate of $2000 \mathrm{~s}^{-1}$. Quasi-static tests are also performed at a strain rate of $0.005 \mathrm{~s}^{-1}$. The specific energy absorption (SEA) is compared under both loading conditions to investigate the performance of such structures under dynamic loading. Results show that both structures exhibit higher SEA values under high deformation rates. In fact, Neovius structures outperform FRD structures in terms of specific energy absorption as it exhibits a SEA value of $22.11 \mathrm{~J} / \mathrm{g}$ and $24.8 \mathrm{~J} / \mathrm{g}$ SEA in quasi-static and dynamic conditions, respectively.
\end{abstract}

\section{Introduction}

Recently, nature-inspired triply periodic minimal surfaces (TPMS) have attracted significant attention due to their superior mechanical properties. Such lightweight structures can be potential candidates for applications in the fields of automotive, aerospace and military.

The mechanical properties of TPMS lattice structures have been extensively studied in literature under quasi-static loadings. A study by Maskery et al. [1] explored the energy absorption of Gyroid TPMS structures under quasi-static compression. Lee et al. [2] numerically explored the mechanical properties of Shwarz Primitive topology under quasi-

* Corresponding author: Sara.AlMahri@tii.ae 
static loading. The authors concluded that TPMS lattices usually exhibit stretch-dominated deformation, which enhances their strength to weight ratio. Studies by Al-Ketan [3-5] showed that diamond lattices exhibit superior compressive modulus and strength when compared by other topologies. However, the mechanical performance of TPMS lattices have not been explored under dynamic loading.

Previous studies have utilized the Split Hopkinson Pressure Bar (SHPB) to characterize bulk material under high strain rates [6-8]. Nonetheless, such method is associated with several challenges when employed to test cellular lattice structures. Therefore, researchers have explored alternative method called Direct Impact Hopkinson bar (DIHB) through modifying the experimental approach [9-13].

Thus, this study aims to explore the mechanical performance of FRD and Neovius TPMS lattices under dynamic through an experimental approach. A Direct Impact Hopkinson Bar (DIHB) is used to perform impact compression tests on the fabricated specimens. The specific energy absorption (SEA) is then evaluated and compared under both loading conditions.

\section{Materials and Methods}

\subsection{Architectural Design}

TPMS lattices can be generated through mathematically approximation methods. In this study, the c-level set method is employed to generate computer-aided design (CAD) models of FRD and Neovius lattices such that $\emptyset=0$. The surfaces of the lattices are described by the following equations:

$$
\begin{gathered}
\phi_{F R D}(x, y, z)=4(\cos X \cos Y \cos Z) \\
-(\cos 2 X \cos 2 Y+\cos 2 Y \cos 2 Z+\cos 2 Z \cos 2 X) \\
\phi_{\text {Neovius }}(x, y, z)=3(\cos X+\cos Y+\cos Z)+4(\cos X \cos Y \cos Z)
\end{gathered}
$$

As shown in Fig. 1, the unit cell is designed to be a cubic of a side length of $4 \mathrm{~mm}$. The lattices are made of an array of $4 \times 4 \times 4$ tessellating unit cells generating a $16 \mathrm{~mm} \times 16 \mathrm{~mm} \times 16 \mathrm{~mm}$ lattice structure. Both structures are designed at a relative density $(\bar{\rho})$ of $30 \%$, where $\bar{\rho}=\rho_{\text {Lattice }} / \rho_{\text {solid }}$. The 3D CAD models are then converted to compatible STL files ready for printing.

\subsection{Experimental Methods}

\subsubsection{Additive Manufacturing of TPMS Lattices}

The fabrication of the two lattice structures was carried out on an SLM machine (EOS M290, $\mathrm{GmbH}$, Munich, Germany). The lattices were fabricated out of gas-atomized stainless steel (SS316L). The chemical composition of the stainless steel powder is shown in Table 1. The microtopography of the powder is shown in the scanning electron microscopy images taken in Fig. 2. The powder shows a nearly spherical shape of particles. The laser power of the SLM machine was set to $350 \mathrm{~W}$, the build platform temperature was $160{ }^{\circ} \mathrm{C}$, and a layer thickness of $40 \mu \mathrm{m}$ is used to fabricate the specimen. A post heat treatment is performed in a 
vacuum furnace for 2 hours at a temperature of $600{ }^{\circ} \mathrm{C}$ to reduce the thermal stresses generated during the SLM process.

a)

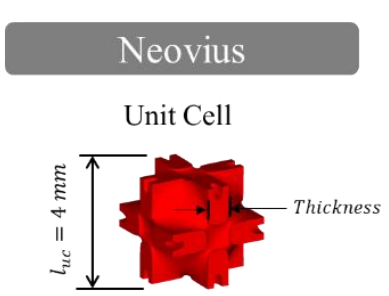

Lattice

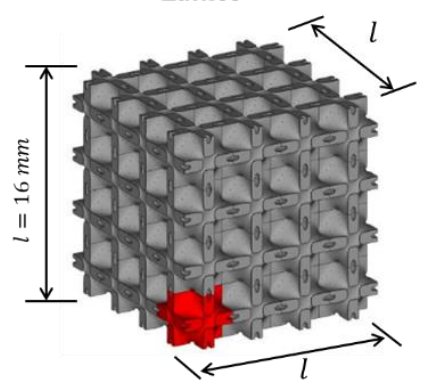

b)

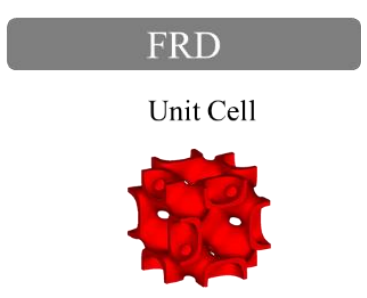

Lattice

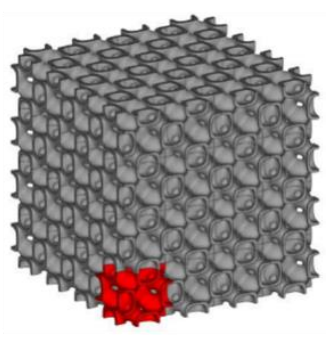

Fig. 1. 3D CAD models of single unit cell and lattice structures of a) Neovius and b) FRD TPMS topologies.

Table 1. Chemical composition of the powder used to fabricate the TPMS lattices (SS316L).

\begin{tabular}{llllllllll}
\hline Element & $\mathrm{C}$ & $\mathrm{Si}$ & $\mathrm{Mn}$ & $\mathrm{Cr}$ & $\mathrm{Ni}$ & $\mathrm{Mo}$ & $\mathrm{P}$ & $\mathrm{S}$ & $\mathrm{Fe}$ \\
\hline Wt. $\%$ & $0-0.03$ & $0-1$ & $0-2$ & $16-18$ & $10-12$ & $2-3$ & $0-0.045$ & $0-0.03$ & Balance \\
\hline
\end{tabular}

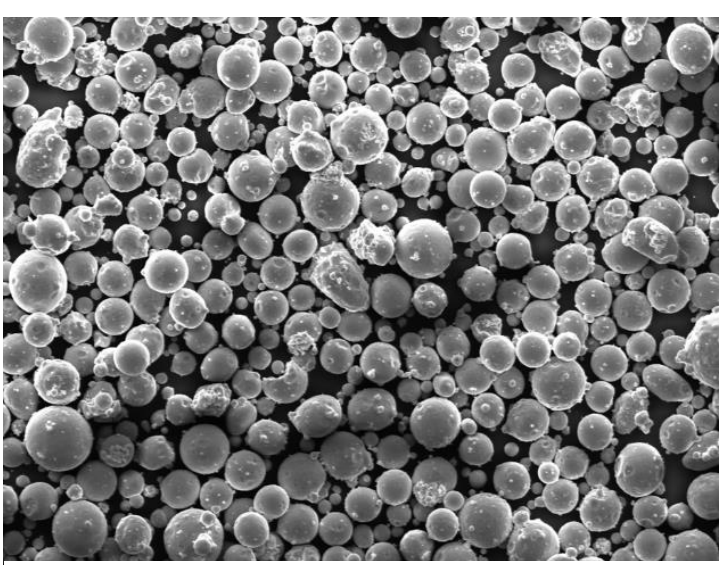

Fig. 2. SEM image of the size and shape distribution of stainless steel (SS316L) 


\subsubsection{Microstructural Characterizaiton}

After fabrication, microstructural characterization was conducted using a scanning electron micro- scope (Quanta ${ }^{\mathrm{TM}} 250 \mathrm{FEG}$ ) to assess the quality the printed specimens. To evaluate the total volume occupied by the specimen, the actual relative density of the fabricated specimen was measured using the dry weighing method. The designed and the actual relative densities were then compared, and the deviation was reported.

\subsubsection{Quasi-Static Testing}

The quasi-static mechanical response of the fabricated specimens was captured through performing a uniaxial compression test using a universal testing machine (MTS E45). The specimens were tested under displacement control compression at a strain rate of $0.005 \mathrm{~s}^{-1}$. The compressive load was measured with a $300 \mathrm{kN}$ load cell, and the displacement was recorded by the crosshead vertical movement. All lattices were compressed up to full densification or until the recorded force reached $285 \mathrm{kN}$. The obtained load-displacement curves were then converted into stress-strain curves taking into consideration the initial dimensions of the specimens. Each test was performed at least three times to confirm repeatability. The plateau stress and the specific energy absorption (SEA) were further evaluated and analyzed.

\subsubsection{DIHB Impact Testing}

The dynamic performance of the printed lattices was investigated through performing a direct impact Hopkinson bar (DIHB) test. The samples were attached to the end of the transmitted bar while a striker is accelerated to impact the specimen, as shown in Fig. 3. The bar and the striker are made out of SAE 1055 steel, with Young's modulus of $193.91 \mathrm{MPa}$, density of $7816.1 \mathrm{~kg} / \mathrm{m}^{3}$, diameter of $25 \mathrm{~mm}$, and a length of $2 \mathrm{~m}$. A $150 \mathrm{~mm}$ length striker with a mass of $0.559 \mathrm{~kg}$ was used for the impact, with a compressed air reservoir attached to a high speed discharge valve. The transmitted bar signal was recorded with a pair of strain gauges (half-bridge), symmetrically attached $250 \mathrm{~mm}$ away from the specimen bar interface. A Photron Fastcam SA5 high speed camera was used to capture a sequence of images at a rate of 100,000 FPS, from which lattices strain values and striker velocity were extracted through an image tracking via PFA software (See Fig. 3 ). The impact velocity was set to $33 \mathrm{~m} / \mathrm{s}$ for all the sample tested.

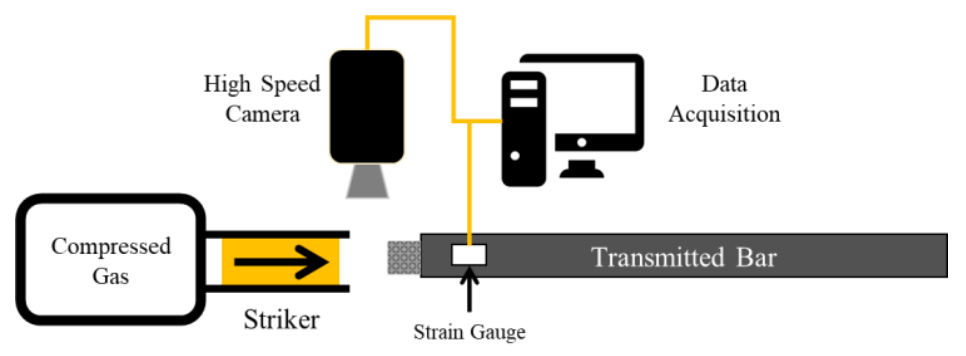

Fig. 3. Schematic of the direct impact Hopkinson bar (DIHB) setup used to test the TPMS lattices 


\section{Results and Discussion}

\subsection{Microstructural Assessment}

After fabrication, the actual relative density of the printed samples was measured using the air weighing method. Table 1 shows the deviation between the designed and the actual relative density. It is observed that the actual relative density of both lattices are lower than the designed values. Neovius and FRD lattices reported a deviation of $8.86 \%$ and $7.5 \%$, respectively. Such deviations can be attributed to the presence of microstructural defects and voids in the printed specimens. Fig. 4 shows the SEM images taken for Neovius and FRD structures. The images show that printing imperfections exist, and some microstructural defects are contributing to the reduction observed in the total volume occupied by the material. The observed voids and defects may be attributed to the solidification process that is associated with the laser additive manufacturing.

Table 2. The deviation between the designed and measured actual relative density of the printed samples.

\begin{tabular}{lccc}
\hline Lattice Topology & Designed RD (\%) & Weighted RD (\%) & Deviation (\%) \\
\hline Neovius & 30 & 27.34 & 8.86 \\
\hline FRD & 30 & 27.75 & 7.5 \\
\hline
\end{tabular}
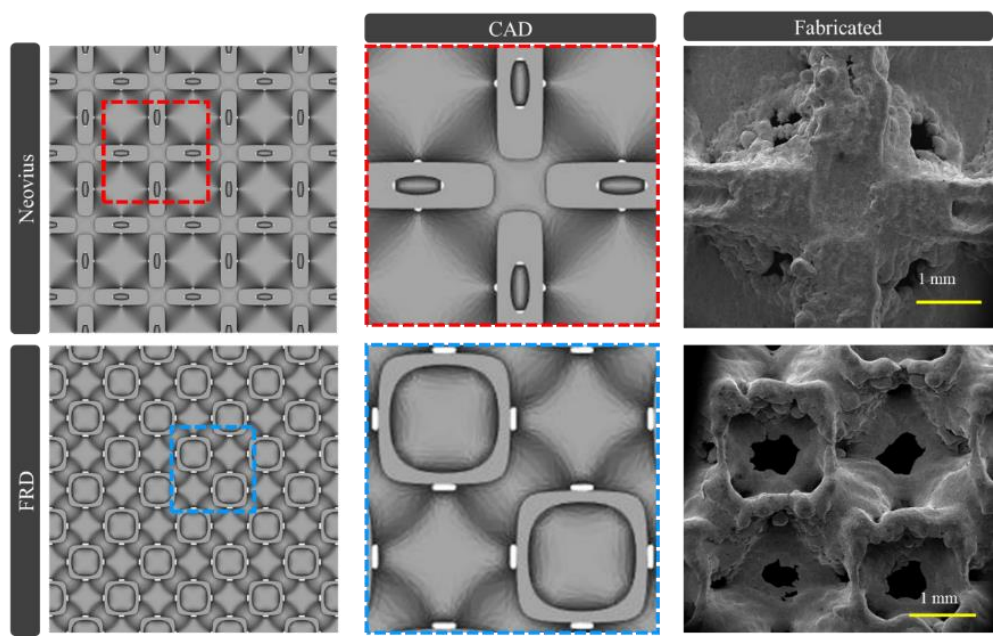

Fig. 4. SEM images of Neovius and FRD showing the printing quality of the printed specimens. 


\subsection{Effect of Dynamic Loading on Mechanical Performance}

In this section, the dynamic performance of Neovius and FRD TPMS lattices is explored. Fig. 5 shows the mechanical stress strain response of both lattices tested under quasi-static and dynamic loading conditions. It is clear that both lattices exhibit enhanced mechanical properties as they undergo dynamic loading. To better understand the mechanical performance, the plateau stress, and the specific energy absorption was evaluated and reported in Figs. 6 and 7.
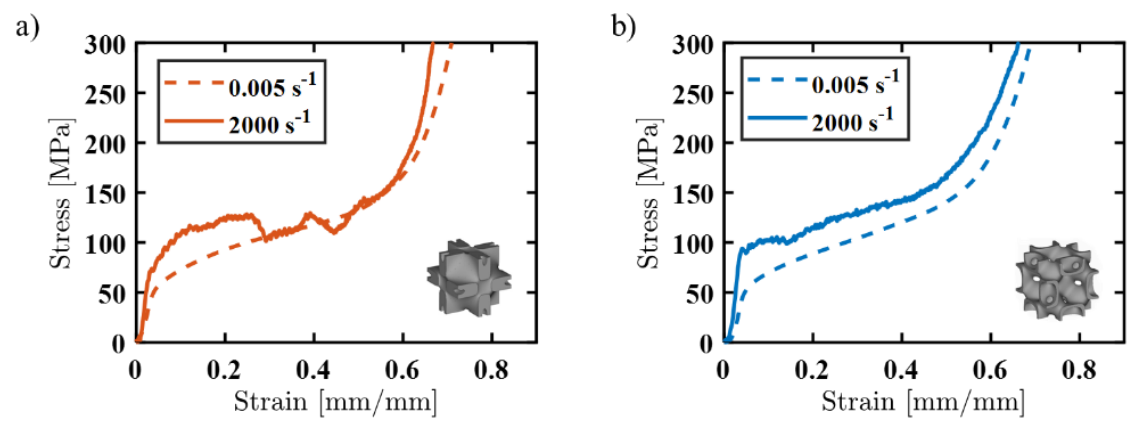

Fig. 5. Quasi-static and dynamic stress-strain response of a) Neovius and b) FRD.

Fig. 6 shows the plateau stress calculated for Neovius under quasi-static and dynamic conditions. An overall trend is observed such that both structures exhibit higher values of plateau stress as they experience higher deformation rates. This enhancement is due to the strain hardening effects. Results show that although both structures show relatively similar values of plateau stress, Neovius exhibits higher plateau stress when compared to FRD structure, under both loading conditions. Neovius structure shows a plateau stress of $102 \mathrm{MPa}$ and $118 \mathrm{MPa}$ in quasi-static and dynamic compression, consequently. Under quasi-static compression, FRD shows a plateau stress of $94.15 \mathrm{MPa}$. In fact, it displays an increase of $19.5 \%$ in plateau stress levels as it undergoes dynamic loading.

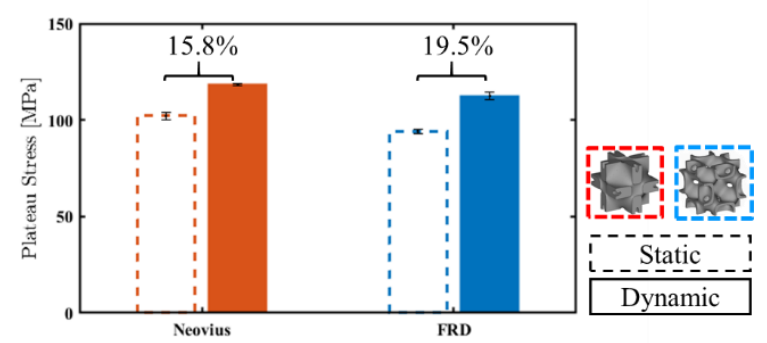

Fig. 6. Plateau stress of Neovius and FRD TPMS lattices under static and dynamic loading. 
Furthermore, the SEA of both lattices is evaluated. SEA is one of the most critical parameters in energy absorption applications. In this study, SEA is evaluated by the following equation [14] :

$$
S E A=\int_{0}^{\delta} \frac{F d \delta}{m}=\frac{\int_{0}^{\varepsilon} \sigma(\varepsilon) d \varepsilon}{\bar{\rho}}
$$

As $\mathrm{F}$ is the compressive force applies, $\mathrm{m}$ is the mass and $\bar{\rho}$ is the relative density. The SEA is calculated up to full densification of each of the structures. Fig. 7 shows the SEA values obtained for both Neovius and FRD under both loading conditions. Neovius shows superior SEA capability when compared to FRD as it exhibits a $22.11 \mathrm{~J} / \mathrm{g}$ and $24.8 \mathrm{~J} / \mathrm{g}$ SEA in quasistatic and dynamic conditions, respectively. This is equivalent to an increase of $12.4 \%$. Moreover, FRD shows an enhancement of $13.9 \%$ in SEA values. Similar to what is observed in plateau stress, the SEA ability of TPMS lattices increases as they experience dynamic loadings. This increase is also attributed to strain hardening effects. As strain rate increase, structures show higher levels of plateau stress, which eventually increases the area under the stress-strain curve. This contributes significantly to the enhancement observed in the SEA values.

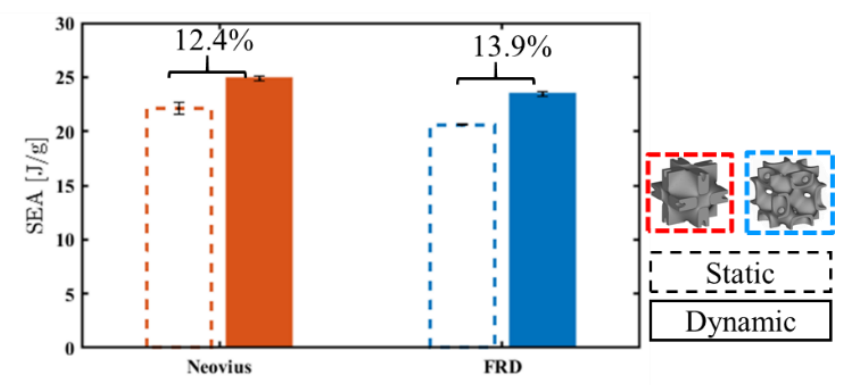

Fig. 7. SEA of Neovius and FRD TPMS lattices under static and dynamic loading.

\section{Conclusions}

In this study, two TPMS lattices were designed, fabricated, and tested under dynamic loading conditions. The fabricated samples were additively manufactured from stainless steel 316L. The quality of the printed structures was assessed through conducting scanning electron microscopy (SEM). The printed samples were tested under dynamic loading using a direct impact Hopkinson bar (DIHB) and quasi-static loading using a UTM. The effects of dynamic loading on the mechanical performance of both structures are explored. From the carried-out tests, the following conclusions are made:

- Both structures exhibit enhanced plateau stress values as they undergo dynamic loading. Neovius exhibits the higher plateau stress values than FRD under both loading conditions.

- Higher values of SEA and Plateau stress are observed under higher deformation rates due to strain hardening effects. 
- Neovius shows superior SEA performance when compared to FRD. 


\section{References}

1. I Maskery, N T Aboulkhair, A O Aremu, C J Tuck, and I A Ashcroft. Additive Manufacturing, 16:24-29, (2017).

2. Dong Wook Lee, Kamran A. Khan, and Rashid K. Abu Al-Rub. International Journal of Plasticity, 95:1-20, (2017).

3. Oraib Al-Ketan, Reza Rowshan, and Rashid K. Abu Al-Rub. Additive Manufacturing, 19:167-183, (2018).

4. Oraib Al-Ketan and Rashid K. Abu Al-Rub. Advanced Engineering Materials, 21(10):139, (2019).

5. Oraib Al-Ketan, Dong Wook Lee, Reza Rowshan, and Rashid K. Abu Al-Rub. Journal of the Mechanical Behavior of Biomedical Materials, 102 (October 2019):103520, (2020).

6. Howard Kuhn and Dana Medlin, editors. ASM Interna-tional, (2000).

7. Muhammad A Kariem, Rafael C Santiago, Reuben Govender, Dong Wei Shu, Dong Ruan, Ger- ald Nurick, Marcilio Alves, Guoxing Lu, and Genevieve S Langdon. International Journal of Impact Engineering, 126:62-75, (2019).

8. Weinong W. Chen and Bo Song. Springer, Boston, MA, (2011).

9. R A Govender and R J CurryJournal of Dynamic Behavior of Materials, 2(1):43-49, (2016).

10. H Zhao, I Elnasri, and S Abdennadher. International Journal of Mechanical Sciences, 47(4- 5):757-774, (2005).

11. Yu Duan, Bing Du, Xiaopeng Shi, Bing Hou, and Yulong Li. International Journal of Impact Engineering, 132:103303, oct 2019.

12. J A Harris, R E Winter, and G J McShane. International Journal of Impact Engineering, 104:177-191, (2017).

13. Pengfei Wang, Songlin Xu, Zhibin Li, Jinglei Yang, Chao Zhang, Hang Zheng, and Shisheng Hu. Materials Science and Engineering: A, 620:253-261, (2015).

14. Xiaofei Cao, Shengyu Duan, Jun Liang, Wei-Bin Wen, and Daining Fang. International Journal of Mechanical Sciences, 145, (2018). 